\title{
The random-cluster model on a homogeneous tree
}

\section{Olle Häggström}

Department of Mathematics, Chalmers University of Technology, S-412 96 Göteborg, Sweden

Received: 28 February 1995/In revised form: 19 July 1995

Summary. The random-cluster model on a homogeneous tree is defined and studied. It is shown that for $1 \leqq q \leqq 2$, the percolation probability in the maximal random-cluster measure is continuous in $p$, while for $q>2$ it has a discontinuity at the critical value $p=p_{c}(q)$. It is also shown that for $q>2$, there is nonuniqueness of random-cluster measures for an entire interval of values of $p$. The latter result is in sharp contrast to what happens on the integer lattice $\mathbf{Z}^{d}$.

Mathematics Subject Classification (1991): 60K35, 82B20, 05C80

\section{Introduction}

The random-cluster model is a two-parameter family of processes living on the edge set of a (finite or infinite) graph. It generalizes independent bond percolation, uniform spanning trees, and Ising/Potts models. It was introduced by Fortuin and Kasteleyn in the early $70 \mathrm{~s}$ in a series of papers $[10,8,9]$. After a period of relative silence, the random-cluster model has enjoyed a revival since the late $80 \mathrm{~s}$, some of the most influential papers being $[1,7,14]$. A nice introduction to the model is given in [13].

Study of the random-cluster model has so far essentially been confined to finite graphs and to graphs embedded in the cubic lattice $\mathbf{Z}^{d}$. The purpose of the present paper is to find out what happens if we let the process take place on an infinite homogeneous tree. It turns out that the most immediate way to define the random-cluster model on such a tree yields nothing new. We find a different way of defining it, viewing the tree as being "connected at infinity", which does lead to interesting phenomena. The main problems we attack are the nature of the onset of an infinite cluster as the parameters vary, and the question of (non-)uniqueness of random-cluster measures for given parameters.

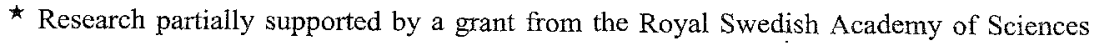


The definition of a random-cluster measure is easiest for a finite graph, so we begin with this case. Let $G$ be a finite graph with vertex set $V$ and edge set $E$. By a subgraph of $G$ we here mean a graph with the same vertex set $V$ as $G$ and an edge set which is a subset of $E$. A subgraph of $G$ is identified with an element of $\{0,1\}^{E}$, where a 1 indicates that an edge is present and a 0 indicates that it is absent.

Definition 1.1 Let $0 \leqq p \leqq 1$ and $q>0$. The random-cluster measure $\mu_{G}^{p, q}$ is the probability measure on the set of subgraphs of $G$ given by

$$
\mu_{G}^{p, q}(\eta)=\frac{1}{Z_{G}^{p, q}}\left\{\prod_{e \in E} p^{\eta(e)}(1-p)^{1-\eta(e)}\right\} q^{k(\eta)}
$$

for all $\eta \in\{0,1\}^{E}$. Here $k(\eta)$ is the number of connected components of $\eta$ and

$$
Z_{G}^{p, q}=\sum_{\eta \in\{0,1\}^{E}}\left\{\prod_{e \in E} p^{\eta(e)}(1-p)^{1-\eta(e)}\right\} q^{k(\eta)}
$$

is the normalizing constant.

When $q=1$, the factor $q^{k(\eta)}$ disappears and all edges become independent; this corresponds to independent bond percolation. The Ising model corresponds to $q=2$ and Potts models to the cases $q=2,3, \ldots$ (see $[7,13]$ ). A uniform spanning tree measure is obtained by letting $p, q \rightarrow 0$ in such a way that $q / p \rightarrow$ 0 (see [15]).

In the special case where $G$ itself is a tree, $\mu_{G}^{p, q}$ reduces, for any $p$ and $q$, to the $q=1$ situation where all edges are independent. To see this, note that for any $\eta \in\{0,1\}^{E}$ we have

$$
k(\eta)+m(\eta)=|V|=1+|E|,
$$

where $m(\eta)$ is the number of edges present in $\eta$. Hence, for any $\eta$,

$$
\begin{aligned}
\mu_{G}^{p, q}(\eta) & =\frac{1}{Z_{G}^{p, q}} p^{m(\eta)}(1-p)^{|E|-m(\eta)} q^{k(\eta)} \\
& =\frac{(1-p)^{|E|} q^{1+|E|}}{Z_{G}^{p}, q}\left(\frac{p}{(1-p) q}\right)^{m(\eta)}
\end{aligned}
$$

so that $\mu_{G}^{p, q}(\eta)$ is simply product measure with edge density

$$
\frac{p}{p+(1-p) q} \text {. }
$$

Definition 1.1 cannot be applied immediately to infinite graphs, but there are natural generalizations, so called thermodynamic limits. Consider first the nearest-neighbour graph on $\mathbf{Z}^{d}$, i.e. the graph whose vertex set is $\mathbf{Z}^{d}$ and where there is an edge between $x$ and $y$ if and only if their (Euclidean) distance is 1 . Write (with some abuse of notation) $\mathbf{Z}^{d}$ for this graph and $\mathbf{E}^{d}$ for its edge set.

Again pick $p$ and $q$ such that $0 \leqq p \leqq 1$ and $q>0$. For a finite set $S \subset \mathbf{E}^{d}$, let $S^{\prime}$ denote the set $\left\{v \in \mathbf{Z}^{d}: \exists e \in S\right.$ such that $e$ is incident to $\left.v\right\}$. For 
a configuration $\xi \in\{0,1\}^{\mathbf{E}^{d} \backslash S}$ of edges off $S$, let the random-cluster measure $\mu_{S}^{p, q}$ "on $S$ with boundary condition $\xi$ " be given by

$$
\mu_{S, \xi}^{p, q}(\eta)=\frac{1}{Z_{S, \xi}^{p, q}}\left\{\prod_{e \in S} p^{\eta(e)}(1-p)^{1-\eta(e)}\right\} q^{k(\eta, \xi)}
$$

for all $\eta \in\{0,1\}^{S}$, where $Z_{S, \xi}^{p, q}$ is the appropriate normalizing constant as in Definition 1.1 , and $k(\eta, \xi)$ is the number of connected components which intersect $S^{\prime}$ in the configuration which agrees with $\eta$ on $S$ and with $\xi$ on $\mathbf{E}^{d} \backslash S$.

Definition 1.2 A probability measure $\mu$ on $\{0,1\}^{\mathrm{E}^{d}}$ is called a random-cluster measure with parameters $p$ and $q$ if its conditional probabilities satisfy

$$
\mu(\eta \mid \xi)=\mu_{S, \zeta}^{p, q}(\eta)
$$

all finite $S \subset \mathbf{E}^{d}$, all $\eta \in\{0,1\}^{S}$ and $\mu$-a.e. $\xi \in\{0,1\} \mathbf{E}^{d} \backslash S$.

This is analogous to the Dobrushin-Lanford-Ruelle definition of a Gibbs state (see [11]). Notice that this defines a consistent set of conditional probabilities, and that the same conditional probabilities hold for finite graphs. Given any $0 \leqq p \leqq 1$ and $q>0$, there exists at least one random-cluster measure for $\mathbf{Z}^{d}$ with these parameters (see [14]). The question of whether there is in fact a unique random-cluster measure is a very intricate one; we refer to [14] again.

We now turn to the case where the graph on which the random-cluster model takes place is a homogeneous tree. Let $\mathbf{T}_{n}$ be the homogeneous tree of order $n$, i.e. $\mathbf{T}_{n}$ is the (unique) infinite graph which is connected, has no circuits, and has $n+1$ branches emanating from every vertex. We will always assume that $n \geqq 2$; the tree obtained with $n=1$ is simply $\mathbf{Z}$, and most of our results do not apply to this case. Write $\mathbf{V}_{n}$ and $\mathbf{E}_{n}$ for the vertex set and the edge set, respectively, of $\mathbf{T}_{n}$. Designate one of the vertices of $\mathbf{T}_{n}$ as the root, and denote it $\mathbf{0}$. For an edge $e \in \mathbf{E}_{n}$, let $|e|$ denote the length of the shortest path starting with $e$ and ending at $\mathbf{0}$. For two edges $e_{1}$ and $e_{2}$ we write $e_{1}<e_{2}$ if $e_{1} \neq e_{2}$ and $e_{1}$ is located on the shortest path from $e_{2}$ to 0 , otherwise we write $e_{1} \nless e_{2}$. If $e_{1}<e_{2}$ we call $e_{2}$ a descendant of $e_{1}$. If $e_{1}<e_{2}$ and $\left|e_{2}\right|=\left|e_{1}\right|+1$, we call $e_{2}$ a child of $e_{1}$, and $e_{1}$ a parent of $e_{2}$. Finally, call two edges siblings if either they have the same parent or they are both incident to $\mathbf{0}$.

Now we could define a random-cluster measure on $\mathbf{T}_{n}$ in the exact same way as a random-cluster measure on $\mathbf{Z}^{d}$. It is easy to see, however, that such a measure will inherit the independence property of random-cluster measures on finite trees. Thus, this would only be a complicated way of defining independent bond percolation on a tree, and we would lose the "essence" of the randomcluster model. To capture this essence, we propose a different definition of a random-cluster model on $\mathbf{T}_{n}$.

Given $S \subset \mathbf{E}_{n}$, define $S^{\prime} \subset \mathbf{V}_{n}$ analogously to the $\mathbf{Z}^{d}$ case. For a configuration $\xi \in\{0,1\}^{\mathbf{E}_{n} \backslash S}$, let

$$
\mu_{S, \check{\zeta}}^{p, q}(\eta)=\frac{1}{Z_{S, \xi}^{p, q}}\left\{\prod_{e \in S} p^{\eta(e)}(1-p)^{1-\eta(e)}\right\} q^{k^{*}(\eta, \xi)}
$$


for all $\eta \in\{0,1\}^{S}$, where $k^{*}(\eta)$ is the number of finite connected components which intersect $S^{\prime}$ in the configuration which agrees with $\eta$ on $S$ and with $\xi$ on $\mathbf{E}_{n} \backslash S$, and $Z_{S, \xi}^{p, q}$ of course again is the right normalizing constant.

Definition 1.3 A probability measure $\mu$ on $\{0,1\}^{\mathbf{E}_{n}}$ is called a random-cluster measure with parameters $p$ and $q$ if its conditional probabilities satisfy

$$
\mu(\eta \mid \xi)=\mu_{S, \xi}^{p, q}(\eta)
$$

for all finite $S \subset \mathbf{E}_{n}$, all $\eta \in\{0,1\}^{S}$ and $\mu$-a.e. $\xi \in\{0,1\}^{\mathbf{E}_{n} \backslash S}$. Here $\mu_{S, \xi}^{p, q}(\eta)$ is given by (1).

This set of conditional probabilities is also consistent. This different way of counting connected components is equivalent to viewing all infinite components as a single component connected at infinity. If we were to apply this definition on $\mathbf{Z}^{d}$, we would most likely retrieve the usual random-cluster model, since it is believed that any random-cluster measure on $\mathbf{Z}^{d}$ has a.s. at most one infinite cluster (if we restrict to translation invariant measures this is certainly the case, by an application of the Burton-Keane uniqueness theorem [5]).

The first thing we would like to prove is the existence of at least one random-cluster measure for this model (given $p$ and $q$ ). Some care is needed, as the usual compactness argument used to prove existence of Gibbs measures (see [11]) does not quite work here. To see what can go wrong, consider the following example. Let $S_{1}, S_{2}, \ldots$ be an increasing sequence of finite subsets of $\mathbf{E}_{n}$ converging to $\mathbf{E}_{n}$ in the sense that each $e \in \mathbf{E}_{n}$ is in all but finitely many $S_{i}$. For each $i$, let $\mu_{i, \xi_{0}}^{p, q}$ be the random-cluster measure on $S_{i}$ with boundary condition $\xi_{0} \equiv 0$ on $\mathbf{E}_{n} \backslash S_{i}$. Since there will then be no infinite clusters, we get, as for finite trees,

$$
\mu_{i, \xi_{0}}^{p, q}(\eta)=\prod_{e \in S_{i}}\left(\frac{p}{p+(1-p) q}\right)^{\eta(e)}\left(\frac{(1-p) q}{p+(1-p) q}\right)^{1-\eta(e)}
$$

for all $\eta \in\{0,1\}^{S_{i}}$. We get weak convergence (in the usual product topology) to a limiting measure $\mu$ which will be product measure with edge probability $p(p+(1-p) q)^{-1}$. Now pick $p$ and $q$ such that

$$
\frac{p}{p+(1-p) q}>\frac{1}{n} \text {. }
$$

A simple branching process argument then shows that $\mu$ will assign positive probability to the event that a given edge $e \in \mathbf{E}_{n}$ lies in an infinite cluster $C$ in such a way that $C$ falls apart into two infinite clusters if $e$ is removed. It is now easy to see that $\mu$ cannot satisfy Definition 1.3 when $q \neq 1$.

Fortunately, we have existence nevertheless, as stated in the following Proposition, which will be proved by a method quite different from the compactness arguments referred to above.

Proposition 1.4 Given $p \in[0,1], q>0$, and a homogeneous tree $\mathbf{T}_{n}$, there exists at least one measure $\mu$ on $\{0,1\}^{\mathbf{E}_{n}}$ which satisfies Definition 1.3.

When $q \geqq 1$, certain monotonicity and correlation inequalities are in force (see Sections 3 and 4 ) so that it makes sense to speak of a maximal measure $\mu_{1}^{p, q}$, which dominates all other random-cluster measures with the given 
parameters, and which is also invariant under graph automorphisms of $\mathbf{T}_{n}$. Such a measure is obtained as a weak limit, as above, with boundary condition $\xi_{1} \equiv 1$. This approach to the random-cluster model on trees was first suggested by Chayes et al. [6]. Let $\theta(p, q)$ denote the probability, under $\mu_{1}^{p, q}$, that the root 0 lies in an infinite cluster. We will prove the following theorem (in which $n$ is suppressed; all quantities of course depend on $n$ also).

Theorem 1.5 Given $q \geqq 1$, there exists a critical value $p_{c}(q) \in(0,1)$ such that $\theta(p, q)=0$ when $p<p_{c}(q)$ and $\theta(p, q)>0$ when $p>p_{c}(q)$. For $1 \leqq q \leqq$ 2 ,

$$
p_{c}(q)=\frac{q}{n+q-1}
$$

while for $q>2$,

$$
p_{c}(q)<\frac{q}{n+q-1} .
$$

We will also show that $p_{c}(q)$ for $q>2$ can be characterized as the unique value of $p \in(0,1)$ for which the polynomial

$$
(q-1) x^{n+1}+\left(1-\frac{p}{1-p}-q\right) x^{n}+\left(\frac{p}{1-p}+1\right) x-1
$$

has a double root in $(0,1)$. For $n=2$, this implies that

$$
p_{c}(q)= \begin{cases}\frac{q}{q+1} & \text { if } 1 \leqq q \leqq 2 \\ \frac{2 \sqrt{q-1}}{1+2 \sqrt{q-1}} & \text { if } q \geqq 2 .\end{cases}
$$

The next theorem, in which $q$ should be thought of as fixed and $\theta(p, q)$ as a function of $p$ only, gives further qualitative description of $\theta(p, q)$.

Theorem 1.6 For $q \geqq 1, \theta(p, q)$ is nondecreasing in $p$. For $1 \leqq q \leqq 2$, $\theta(p, q)$ is continuous for all $p$. For $q>2, \theta(p, q)$ is continuous for all $p \neq p_{c}(q)$, while at $p=p_{c}(q), \theta(p, q)$ is continuous from the right but not from the left.

Hence,

$$
\theta\left(p_{c}(q), q\right) \begin{cases}=0 & \text { for } 1 \leqq q \leqq 2 \\ >0 & \text { for } q>2\end{cases}
$$

In physics language, we say that we have a first order phase transition for $q>2$ while only a second order phase transition for $1 \leqq q \leqq 2$. This phenomenon was observed in [6] for $n=2$. It is interesting to compare our results with those in [4], where a critical phenomenon at $q=2$ is also reported; this time in the asymptotics of random-cluster models on large (but finite) complete graphs.

The next issue we would like to address is the question of uniqueness of random-cluster measures. Let $p_{c}(q)$ be as in Theorem 1.5 . We will prove

Theorem 1.7 Let $q>2$. Then there is more than one random-cluster measure for every value of $p$ in the interval

$$
\left[p_{c}(q), \frac{q}{n+q-1}\right] \text {. }
$$


This is in sharp contrast to the situation on the integer lattice $\mathbf{Z}^{d}$, where it is known that for each $q \geqq 1$, there is nonuniqueness for at most countably many values of $p$, as shown in [14]. Perhaps the unexpectedness of this contrasting behaviour drops a little bit if one considers the following. The method used in [14] to prove uniqueness for all but at most countably many $p$ is the same type of convexity arguments which were used in [17] to show that the Ising model on $\mathbf{Z}^{d}$ with non-zero external field has a unique Gibbs measure. This latter result turned out to fail on a tree (see [20]) and Theorem 1.7 might be viewed as an analogous phenomenon.

We can also show uniqueness for some parameter values. The next Theorem tells us that, given $q$, picking $p$ sufficiently small guarantees a unique infinite cluster. The reason is simple: if $p$ is sufficiently small, then there will not be enough edges around to create an infinite cluster, and we will be back in the i.i.d. situation.

Theorem 1.8 There is a unique random-cluster measure if either

$$
q>2 \text { and } p<p_{c}(q)
$$

or

$$
q \leqq 2 \text { and } p \leqq \frac{q}{n+q-1}
$$

There is a big gap between Theorems 1.7 and 1.8 where we can neither establish uniqueness, nor nonuniqueness. We believe that Theorem 1.7 is sharp, i.e. that we have uniqueness for all values of $(p, q)$ in this gap:

Conjecture 1.9 For $q \leqq 2$ there is a unique random-cluster measure for any p. For $q>2$ there is a unique random-cluster measure whenever

$$
p \notin\left[p_{c}(q), \frac{q}{n+q-1}\right] \text {. }
$$

The main technique used in this paper is the construction of a kind of treeindexed Markov chain, which can be embedded into certain random-cluster measures. The random-cluster measures themselves are very non-Markov, because the conditional probability that an edge $e$ is present given the configuration on $\mathbf{E}_{n} \backslash\{e\}$ may depend on edges arbitrarily far away from $e$. The embedded process will contain enough extra information as to yield a Markov structure. This technique will provide a very explicit way to construct randomcluster measures.

A few other references, not mentioned above, deserve to be pointed out here due to the close connections between the random-cluster model and Ising / Potts models (and also percolation). Some papers, in addition to [20], where the Ising model on trees is studied are $[16,18,3]$. An analysis of the $q=3$ Potts model on a binary tree can be found in [21]. Two of the main references for percolation on trees are $[12,19]$.

The rest of this paper is organized as follows. Our tree-indexed Markov chain is constructed in Sect. 2 and used in Sect. 3 to prove Proposition 1.4, Theorems 1.5-1.8, and a small result in support of Conjecture 1.9. In Sect. 4, we discuss what happens to the FKG inequality in our context, and in Sect.5, we show that under the conditions of Theorem 1.7 
there are uncountably many (non-translation invariant) extremal random-cluster measures.

\section{The tree-indexed Markov chain}

In this section we introduce a special type of tree-indexed Markov chain which will be shown to be closely related to random-cluster measures. Our Markov chain will differ slightly from those discussed previously in the literature (e.g. $[2,20,21]$ ) in two respects. First, it is indexed by the edge set $\mathbf{E}_{n}$ of $\mathbf{T}_{n}$ (rather than the vertex set). Second, it exhibits a kind of sibling dependencies which were not allowed for in earlier studies.

The Markov chain has two parameters $b, c \in(0,1)$ which will determine a probability measure $M^{b . c}$ on $\left\{0^{\prime}, 1^{\prime}, 2^{\prime}\right\}^{\mathbf{E}_{n}}$. Here $\left\{0^{\prime}, 1^{\prime}, 2^{\prime}\right\}$ is the state space of the Markov chain. The relation between $M^{b, c}$ and random-cluster measures is via the function $\psi:\left\{0^{\prime}, 1^{\prime}, 2^{\prime}\right\} \rightarrow\{0,1\}$ given by

$$
\begin{aligned}
& \psi\left(0^{\prime}\right)=0, \\
& \psi\left(1^{\prime}\right)=1, \\
& \psi\left(2^{\prime}\right)=1 .
\end{aligned}
$$

We interpret $\psi:\left\{0^{\prime}, 1^{\prime}, 2^{\prime}\right\}^{\mathbf{E}_{n}} \rightarrow\{0,1\}^{\mathbf{E}_{n}}$ as pointwise $\psi$, and let $\psi$ also denote the induced mapping from probability measures on $\left\{0^{\prime}, 1^{\prime}, 2^{\prime}\right\}^{\mathbf{E}_{n}}$ to probability measures on $\{0,1\}^{\mathbf{E}_{n}}$. Proposition 2.2 below states that $\psi\left(M^{b_{*} c}\right)$ is a randomcluster measure.

We write $\xi \in\left\{0^{\prime}, 1^{\prime}, 2^{\prime}\right\}^{\mathbf{E}_{n}}$ for a realization of the Markov chain. For $e \in \mathbf{E}_{n}$, we call $\xi(e)$ the value of $e$. The state space should be thought of as follows. The value $0^{\prime}$ at $e$ means that $e$ is absent in the random-cluster model, while both $1^{\prime}$ and $2^{\prime}$ mean that the edge is present. The difference between $1^{\prime}$ and $2^{\prime}$ is that a $2^{\prime}$ signifies that there is an infinite path of present edges starting at $e$ and after that using descendants of $e$ only (in other words, the path should "radiate outwards" from $e$ as seen from the root). A $1^{\prime}$ signifies that there is no such path of present edges.

In order to describe $M^{b, c}$ more precisely, the following definition is convenient.

Definition 2.1 Let $X$ be a random variable taking values on $\{0,1, \ldots, n\}$ for some $\mathrm{n}$. We say that $X$ has the modified binomial distribution with parameters $n, p \in[0,1]$ and $\theta \in[0,1]$, and write $X \sim \operatorname{Bin}^{*}(n, p, \theta)$ if

$$
X= \begin{cases}0 & \text { w.p. } \theta \\
i & \text { w.p. } \frac{1-\theta}{1-(1-p)^{3}}\left(\begin{array}{c}
n \\
i
\end{array}\right) p^{i}(1-p)^{n-i} \text { for } i \in\{1, \ldots, n\}\end{cases}
$$

In other words, a coin with heads-probability $\theta$ is tossed, and if heads comes up, then $X=0$, while if tail comes up, $X$ gets the binomial $(n, p)$ distribution conditioned to be non-zero. 
The chain is started from the root $\mathbf{0}$. There are $n+1$ edges leading out of 0. Of these, $Y_{2}$ edges take the value $2^{\prime}$, where

$$
Y_{2} \sim \operatorname{Bin}^{*}\left(n+1, b, \frac{(1-c)(1-b)\left(1-(1-b)^{n}\right)}{c b+(1-b)\left(1-(1-b)^{n}\right)}\right) .
$$

These are drawn uniformly from the $n+1$ edges. Among the $n+1-Y_{2}$ remaining edges, each one gets the value $1^{\prime}$ with probability $a$, defined by

$$
a=\frac{b(1-b)^{n-1}}{1-(1-b)^{n}}
$$

and $0^{\prime}$ with probability $1-a$, independently of everything else.

The rest of the realization is built up inductively as follows. The children $e_{1}, \ldots, e_{n}$ of an edge $e$ are assigned values in such a way that, conditionally on the value of $e$, they are independent of the values on the set $\left\{e^{\prime}: e \nless e^{\prime}\right\}$. Conditional on $\left\{\xi(e)=0^{\prime}\right\}$ (resp. $\left\{\xi(e)=1^{\prime}\right\}$ and $\left\{\xi(e)=2^{\prime}\right\}$ ), the number of children which take the value $2^{\prime}$ is given by $Y_{02}$ (resp. $Y_{12}$ and $Y_{22}$ ), where

$$
Y_{02} \sim \operatorname{Bin}^{*}(n, b, 1-c),
$$

while

$$
Y_{12}=0 \text { a.s. }
$$

and

$$
Y_{22} \sim \operatorname{Bin}^{*}(n, b, 0) .
$$

These are drawn uniformly from the $n$ children. Each of the remaining children is independently assigned the value $1^{\prime}$ with probability $a$ and $0^{\prime}$ with probability $1-a$. This defines (uniquely) the measure $M^{b, c}$ on $\xi \in\left\{0^{\prime}, 1^{\prime}, 2^{\prime}\right\}^{\mathbf{E}_{n}}$.

We are now in a position where we can motivate the interpretations given above of the values $1^{\prime}$ and $2^{\prime}$ at an edge $e$. It follows from the distribution of $Y_{22}$ that if $\xi(e)=2^{\prime}$, then a.s. at least one child of $e$ takes the value $2^{\prime}$, and this child must in turn have a child with value $2^{\prime}$, and so on. Hence there a.s. exists an infinite path of edges whose value is $2^{\prime}$ starting at $e$ and moving outwards as seen from the origin.

On the other hand, suppose $\xi(e)=1^{\prime}$. Then a.s. no children of $e$ has value $2^{\prime}$ by the distribution of $Y_{12}$. Therefore the decendants of $e$ that are connected to $e$ via present edges all have value $1^{\prime}$. These edges form a branching process whose offspring distribution is binomial $(n, a)$. We need to show that this branching process is subcritical, i.e. that $n a<1$. To see this, note that

$$
(1-b)^{n-1}(1+(n-1) b) \leqq(1-b)^{n-1}(1+b)^{n-1}=\left(1-b^{2}\right)^{n-1}<1
$$

so that

$$
1-(1-b)^{n}>n b(1-b)^{n-1}
$$

whence

$$
n a=\frac{n b(1-b)^{n-1}}{1-(1-b)^{n}}<1 .
$$

Hence, an edge $e$ for which $\xi(e)=1^{\prime}$ is not connected to infinity via its descendants. 
It is easy to see that the projection of $M^{b, c}$ onto a single self-avoiding path from 0 to infinity is an ordinary ( $Z$-indexed) Markov chain. The ambitious reader may check that this Markov chain is stationary (although this follows directly from Proposition 2.2 below).

The following two Propositions relate our Markov chains to randomcluster measures, and are central to our analysis of random-cluster measures in Section 3. A graph automorphism of $\mathbf{T}_{n}$ is a bijection $\pi: \mathbf{V}_{n} \rightarrow \mathbf{V}_{n}$ such that for $v, w \in \mathbf{V}_{n}$ there is an edge between $\pi(v)$ and $\pi(w)$ if and only if there is an edge between $v$ and $w$.

Proposition $2.2 \psi\left(M^{b, c}\right)$ is a random-cluster measure with parameters

$$
p=\frac{b\left(1-(1-b)^{n}\right)}{b\left(1-(1-b)^{n}\right)+(1-b)\left(1-(1-b)^{n-1}\right) c}
$$

and

$$
q=\frac{\left(1-(1-b)^{n}\right)(1-c)}{(1-b)^{n} c}
$$

Moreover, $\psi\left(M^{b, c}\right)$ is invariant under graph automorphisms of $\mathbf{T}_{n}$.

Proposition 2.3 Let $\mu$ be a probability measure on $\{0,1\}^{\mathbf{E}_{m}}$. Suppose $\mu$ is a random-cluster measure (for some $p$ and $q$ ) and furthermore that $\mu$ is extremal among all random-cluster measures for those parameters. If $\mu$ is also invariant under graph automorphisms of $\mathbf{T}_{n}$, then either

(i) $\mu$ is i.i.d. measure, in which all edges are independently present with probability $p(p+(1-p) q)^{-1}$,

or

(ii) $\mu=\psi\left(M^{b, c}\right)$ for some choice of $b$ and $c$.

The rest of this Section is devoted to proving these results. The following lemma is useful for the proof of Proposition 2.2. Given an edge $e \in \mathbf{E}_{n}$, let $C_{e}$ denote the event that both endvertices of $e$ are connected to infinity via paths not involving $e$, and let $\neg C_{e}$ denote the complement of this event.

Lemma 2.4 Let $p \in(0,1)$ and $q>0$, and let $\mu$ be a probability measure on $\{0,1\}^{\mathbf{E}_{n}}$. If for all $e \in \mathbf{E}_{n}$ and $\mu$-a.e. configuration $\xi \in\{0,1\}^{\mathbf{E}_{n} \backslash\{e\}}$ on the event $C_{e}$ we have

$$
\mu(e \text { is present } \mid \xi)=p
$$

and for $\mu$-a.e. $\xi \in\{0,1\}^{\mathrm{E}_{n} \backslash\{e\}}$ on $\neg C_{e}$ we have

$$
\mu(e \text { is present } \mid \xi)=\frac{p}{p+(1-p) q},
$$

then $\mu$ is a random-cluster measure with parameters $p$ and $q$.

In other words, if we want to check that $\mu$ is a random-cluster measure, then it suffices to check the conditional distributions prescribed by Definition 1.3 for sets consisting of a single edge.

Proof of Lemma 2.4. Suppose the assumptions of the lemma hold. For $e \in \mathbf{E}_{n}$, introduce a Markov process $\zeta_{t}^{e}$ on $\{0,1\}^{\mathbf{E}_{n}}$ as follows. The starting configuration 
$\zeta_{0}^{e}$ is chosen according to $\mu$. For $e^{\prime} \neq e, \zeta_{t}^{e}\left(e^{\prime}\right)$ is kept fixed for all time, while $\zeta_{t}^{e}(e)$ flips (changes its value) at intensity $\gamma\left(\zeta_{t}^{e}\right)$ given by

$$
\gamma\left(\zeta_{t}^{e}\right)= \begin{cases}p & \text { on } C_{e} \cap\{e \text { is absent }\}, \\ 1-p & \text { on } C_{e} \cap\{e \text { is present }\}, \\ \frac{p}{p+(1-p) q} & \text { on } \neg C_{e} \cap\{e \text { is absent }\}, \\ \frac{(1-p) q}{p+(1-p) q} & \text { on } \neg C_{e} \cap\{e \text { is present }\} .\end{cases}
$$

We think of the dynamics as a clock which rings according to a Poisson process with intensity 1 . Each time the clock rings the value at $e$ is forgotten and chosen again according to the conditional probabilities prescribed by the Lemma. Hence, $\zeta_{t}^{e}$ preserves $\mu$ for all $t$.

Now let $S \subset \mathbf{E}_{n}$ be a finite edge-set. Introduce another Markov process $\zeta_{t}^{S}$ on $\{0,1\}^{\mathbf{E}_{n}}$ by choosing $\zeta_{0}^{S}$ according to $\mu$ and letting each $e \in S$ update in the same way as $\zeta_{t}^{e}$ above, and in such a way that the underlying Poisson processes are independent. We see by induction over the total number of Poisson occurencies that $\zeta_{t}^{S}$ preserves $\mu$ for all $t$.

We now condition the process on the configuration off $S$, writing $\zeta_{t}^{S, \xi}$ for the process conditioned on $\zeta_{0}^{S}\left(\mathbf{E}_{n} \backslash S\right)=\xi$. We can view $\zeta_{t}^{S, \xi}$ as a finite state continuous time Markov chain taking values in $\{0,1\}^{S}$. It is easy to see that this Markov chain is irreducible, whence it has a unique stationary distribution. We are done if we can show that this stationary distribution is given by $\mu_{S, \xi}^{p, q}$ (see Definition 1.3). For this, it suffices to show that $\mu_{S, \xi}^{p, q}$ is reversible for $\zeta_{t}^{S, \xi}$, i.e. that for all $\eta, \eta^{\prime} \in\{0,1\}^{S}$,

$$
\mu_{S, \xi}^{p, q}(\eta) \gamma_{\xi}\left(\eta, \eta^{\prime}\right)=\mu_{S, \xi}^{p, q}\left(\eta^{\prime}\right) \gamma_{\xi}\left(\eta^{\prime}, \eta\right)
$$

where $\gamma_{\xi}\left(\eta, \eta^{\prime}\right)$ denotes the rate at which $\eta$ flips to $\eta^{\prime}$. First note that $\gamma_{\xi}\left(\eta, \eta^{\prime}\right)=$ $\gamma_{\xi}\left(\eta^{\prime}, \eta\right)=0$ unless $\eta$ and $\eta^{\prime}$ differ in exactly one $e \in S$. So pick $e \in S$ and $\eta_{0}, \eta_{1} \in\{0,1\}^{S}$ such that $\eta_{0}$ and $\eta_{1}$ are equal except at $e$, where $\eta_{0}(e)=0$ and $\eta_{1}(e)=1$. If $C_{e}$ holds for the configuration which equals $\xi$ on $\mathrm{E}_{n} \backslash S$ and $\eta_{0}$ on $S$, then

$$
\frac{\gamma_{\xi}\left(\eta_{0}, \eta_{1}\right)}{\gamma_{\xi}\left(\eta_{1}, \eta_{0}\right)}=\frac{p}{1-p}=\frac{\mu_{S, \xi}^{p, q}\left(\eta_{1}\right)}{\mu_{S, \xi}^{p, q}\left(\eta_{0}\right)} .
$$

If $C_{e}$ does not hold for this configuration, then

$$
\frac{\gamma_{\xi}\left(\eta_{0}, \eta_{1}\right)}{\gamma_{\xi}\left(\eta_{1}, \eta_{0}\right)}=\frac{p}{(1-p) q}=\frac{\mu_{S, \xi}^{p, q}\left(\eta_{1}\right)}{\mu_{S, \xi}^{p, q}\left(\eta_{0}\right)}
$$

and in either case we have verified (4).

Proof of Proposition 2.2. Let $\mu=\psi\left(M^{b, c}\right)$. We start by proving that $\mu$ is a random-cluster measure. By Lemma 2.4 , we only need to study the conditional distribution of the value $\eta(e)$ at an edge $e$ given $\xi \in\{0,1\}^{\mathbf{E}_{n} \backslash\{e\}}$. Pick $e \in \mathbf{E}_{n}$ and denote its two endvertices $v$ and $w$ in such a way that $v$ is located on the shortest path from $w$ to $\mathbf{0}$ (in the case where $e$ is incident to $\mathbf{0}$ we get $v=\mathbf{0}$ ). Given a configuration $\xi \in\{0,1\}^{\mathbf{E}_{n} \backslash\{e\}}$, define $K_{v}$ to be the set of vertices that 
can be reached from $v$ via paths using edges in the set $\left\{e^{\prime} \in \mathbf{E}_{n} \backslash\{e\}: \zeta\left(e^{\prime}\right)=1\right\}$ only. Define $K_{w}$ analogously, and note that $K_{v}$ and $K_{w}$ are disjoint. We will compute $\mu(\eta(e)=1 \mid \xi)$ in the four cases which arise depending on whether $K_{v}$ or $K_{w}$ are finite or infinite.

Note first that if $\xi\left(\mathbf{E}_{n} \backslash\{e\}\right)$ and $\eta(e)$ are both known, then we can reconstruct the $\left\{0^{\prime}, 1^{\prime}, 2^{\prime}\right\}^{\mathbf{E}_{n}}$-valued configuration corresponding to $\xi$ and $\eta$ (we only need to check, for each present edge, whether it has a path of present edges leading outwards to infinity). Write $\xi^{*}$ for this $\left\{0^{\prime}, 1^{\prime}, 2^{\prime}\right\}^{\mathbf{E}_{n}}$-valued configuration.

Case 1. $K_{v}$ and $K_{w}$ both finite. In this case, we can determine $\xi^{*}$ on $\mathbf{E}_{n} \backslash\{e\}$ without knowledge of $\eta(e)$, because presence of $e$ does not yield any paths outwards to infinity. As a consequence of this, we only need to consider transition probabilities from the parent of $e$ to $e$ and from $e$ to its children in order to calculate the conditional distribution of the value at $e$. We have that $\xi^{*}(e)=0^{\prime}$ (resp. $1^{\prime}$ ) when $\eta(e)=0$ (resp. 1). Let $L_{e}$ denote the set of all edges except for $e$ and its descendants, i.e. $L_{e}=\left\{e^{\prime} \in \mathbf{E}_{n}: e \neq e^{\prime}, e \nless e^{\prime}\right\}$. It follows from the transition probabilities of $M^{b, c}$ that

$$
\frac{M^{b, c}\left(\xi^{*}(e)=1^{\prime} \mid \xi^{*}\left(L_{e}\right)\right)}{M^{b, c}\left(\xi^{*}(e)=0^{\prime} \mid \xi^{*}\left(L_{e}\right)\right)}=\frac{a}{1-a}
$$

for any $\xi^{*}\left(L_{e}\right)$ as long as either of the conditional probabilities is non-zero. Another look at the transition rules shows that the conditional distribution of $\xi^{*}\left(\left\{e^{\prime}: e<e^{\prime}\right\}\right)$ given $\xi^{*}(e)$ depends on $\xi^{*}(e)$ only through the indicator function of the event that none of the children of $e$ takes the value $2^{\prime}$, which is equivalent to the event $\left\{K_{w}\right.$ is finite $\}$. We have

$$
M^{b, c}\left(\left\{K_{w} \text { is finite }\right\} \mid \xi^{*}(e)=i\right)=\left\{\begin{array}{ll}
1-c & \text { for } i=0^{\prime} \\
1 & \text { for } i=1^{\prime}
\end{array} .\right.
$$

Multiplying (5) and (6) yields

$$
\frac{M^{b, c}\left(\xi^{*}(e)=1^{\prime} \mid \xi\left(\mathbf{E}_{n} \backslash\{e\}\right)\right)}{M^{b, c}\left(\xi^{*}(e)=0^{\prime} \mid \xi\left(\mathbf{E}_{n} \backslash\{e\}\right)\right)}=\frac{a}{(1-a)(1-c)}
$$

so that

$$
\begin{aligned}
\mu(\eta(e) & \left.=1 \mid \xi\left(\mathbf{E}_{n} \backslash\{e\}\right)\right)=M^{b, c}\left(\xi^{*}(e)=1^{\prime} \mid \xi\left(\mathbf{E}_{n} \backslash\{e\}\right)\right) \\
& =\frac{a}{a+(1-a)(1-c)} .
\end{aligned}
$$

Case 2. $K_{v}$ infinite, $K_{w}$ finite. By exactly the same reasoning as in Case 1, we obtain the expression (8) for $\mu\left(\eta(e)=1 \mid \xi\left(\mathbf{E}_{n} \backslash\{e\}\right)\right)$.

Case 3. $K_{v}$ finite, $K_{w}$ infinite. This case is slightly more complicated than the previous ones, because now $\xi^{*}$ cannot be completely determined on $\mathbf{E}_{n} \backslash\{e\}$ without knowledge of $\eta(e)$. Let $\left(e_{1}, e_{2}, \ldots, e_{m}, e_{m+1}, \ldots, e_{m+k}, e\right)$ be the edges of the shortest path from 0 ending with $e$. Here $e_{m}$ is the last edge of this path whose value is 0 (if no edge of this path has value 0 , then we take $m=0$ ). So $m$ is random, but in the following computations $e_{m+1}, \ldots, e_{m+k}$ 
will be viewed as a fixed edges. If $\eta(e)=0$, then $\xi^{*}\left(e_{m+1}\right)=\xi^{*}\left(e_{m+2}\right)$ $=\cdots=\xi^{*}\left(e_{m+k}\right)=1^{\prime}$ and $\xi^{*}(e)=0^{\prime}$ while if $\eta(e)=1$, then $\xi^{*}\left(e_{m+1}\right)$ $=\xi^{*}\left(e_{m+2}\right)=\cdots=\xi^{*}\left(e_{m+k}\right)=\xi^{*}(e)=2^{\prime}$. For all other edges $e^{\prime}, \xi^{*}\left(e^{\prime}\right)$ is known (in particular, $\xi^{*}\left(e^{\prime}\right) \neq 2^{\prime}$ for all other children of $e_{m}, \ldots, e_{m+k}$ ). Hence, in order to compute the conditional distribution of the value at $e$, what we need to take into account are the transition probabilities from $e_{m}, \ldots, e_{m+k}$ and $e$. For an edge $e^{\prime}$, let $D\left(e^{\prime}\right)$ denote the event that none of the siblings of $e^{\prime}$ take the value $2^{\prime}$, and note that given the value of its parent, $e^{\prime}$ depends on its siblings only through the indicator of $D\left(e^{\prime}\right)$. Suppose first that $k>0$. The transition rules of $M^{b, c}$ imply that for $m>0$

$$
\frac{M^{b, c}\left(\xi^{*}\left(e_{m+1}\right)=2^{\prime}, D\left(e_{m+1}\right) \mid \xi^{*}\left(e_{m}\right)=0^{\prime}\right)}{M^{b, c}\left(\xi^{*}\left(e_{m+1}\right)=1^{\prime}, D\left(e_{m+1}\right) \mid \xi^{*}\left(e_{m}\right)=0^{\prime}\right)}=\frac{c\left(\frac{b(1-b)^{n-1}}{1-(1-b)^{n}}\right)}{(1-c) a}=\frac{c}{1-c}
$$

while for $m=0$ we get

$$
\frac{M^{b, c}\left(\xi^{*}\left(e_{1}\right)=2^{\prime}, D\left(e_{1}\right)\right)}{M^{b, c}\left(\xi^{*}\left(e_{1}\right)=1^{\prime}, D\left(e_{1}\right)\right)}=\frac{\left(1-\frac{(1-c)(1-b)\left(1-(1-b)^{n}\right)}{b c+(1-b)\left(1-(1-b)^{n}\right)}\right)\left(\frac{b(1-b)^{n}}{1-(1-b)^{n+1}}\right)}{\frac{(1-c)(1-b)\left(1-(1-b)^{n}\right)}{b c+(1-b)\left(1-(1-b)^{n}\right)} a}=\frac{c}{1-c},
$$

where the last equality follows from trivial algebraic manipulation. For $i=$ $2, \ldots, k$ we get

$$
\frac{M^{b, c}\left(\xi^{*}\left(e_{m+i}\right)=2^{\prime}, D\left(e_{m+i}\right) \mid \xi^{*}\left(e_{m+i-1}\right)=2^{\prime}\right)}{M^{b, c}\left(\xi^{*}\left(e_{m+i}\right)=1^{\prime}, D\left(e_{m+i}\right) \mid \xi^{*}\left(e_{m+i-1}\right)=1^{\prime}\right)}=\frac{\frac{b(1-b)^{n-1}}{1-(1-b)^{n}}}{a}=1
$$

while

$$
\frac{M^{b, c}\left(\xi^{*}(e)=2^{\prime}, D(e) \mid \xi^{*}\left(e_{m+k}\right)=2^{\prime}\right)}{M^{b, c}\left(\xi^{*}(e)=0^{\prime}, D(e) \mid \xi^{*}\left(e_{m+k}\right)=1^{\prime}\right)}=\frac{\frac{b(1-b)^{n-1}}{1-(1-b)^{n}}}{1-a}=\frac{a}{1-a}
$$

and

$$
M^{b, c}\left(\left\{K_{w} \text { is infinite }\right\} \mid \xi^{*}(e)=i\right)= \begin{cases}c & \text { for } i=0^{\prime} \\ 1 & \text { for } i=2^{\prime}\end{cases}
$$

similarly to (6). Combining (9)-(13) we get the same expression (7) as in Case 1 , (except that the $1^{\prime}$ in (7) is replaced by a $2^{\prime}$ ) so we have (8) again. The same thing follows when $k=0$ after the necessary modifications of (9)-(12).

Case 4. $K_{v}$ and $K_{w}$ both infinite. As in Case 3, we cannot determine $\xi^{*}\left(\mathbf{E}_{n} \backslash\{e\}\right)$ without knowledge of $\eta(e)$. Let $\left(e_{1}, e_{2}, \ldots, e_{m}, e_{m+1}, \ldots, e_{m+k}, e\right)$ be the edges of the shortest path from $\mathbf{0}$ ending with $e$, where this time $e_{m}$ is the last edge of this path which has a child outside the path whose value is $2^{\prime}$ (this can be determined without knowing $\eta(e)$ ). We may have $k=0$. If $\eta(e)=0$, then $\xi^{*}\left(e_{m+i}\right)=1^{\prime}$ for $i=1, \ldots, k$ and $\xi^{*}(e)=0^{\prime}$, while if $\eta(e)=1$, then $\xi^{*}\left(e_{m+i}\right)=2^{\prime}$ for $i=1, \ldots, k$ and $\xi^{*}(e)=2^{\prime}$. For all other edges, $\xi^{*}$ can be determined without knowing $\eta(e)$. Hence, it is the transitions from $e_{m}, e_{m+1}, \ldots, e_{m+k}$ and $e$ we need to consider. Suppose first that $k>0$. Note 
that $\xi^{*}\left(e_{m}\right)=0^{\prime}$ or $2^{\prime}$, and in either case

$$
\frac{M^{b, c}\left(\xi^{*}\left(e_{m+1}\right)=2^{\prime} \mid \xi^{*}\left(L_{e_{m+1}}\right)\right)}{M^{b, c}\left(\xi^{*}\left(e_{m+1}\right)=1^{\prime} \mid \xi^{*}\left(L_{e_{m+1}}\right)\right)}=\frac{b}{(1-b) a}
$$

by the transition probabilities for $M^{b, c}$. For the transitions from $e_{m+1}, \ldots, e_{m+k}$ and $e$ we recover (11)-(13) from Case 3. Hence, by combining (11)-(14), we get

$$
\frac{M^{b, c}\left(\xi^{*}(e)=2^{\prime} \mid \xi\left(\mathbf{E}_{n} \backslash\{e\}\right)\right)}{M^{b, c}\left(\xi^{*}(e)=0^{\prime} \mid \xi\left(\mathbf{E}_{n} \backslash\{e\}\right)\right)}=\frac{b}{(1-b)(1-a) c}
$$

whence

$$
\begin{aligned}
\mu\left(\eta(e)=1 \mid \xi\left(\mathbf{E}_{n} \backslash\{e\}\right)\right) & =M^{b, c}\left(\xi^{*}(e)=2^{\prime} \mid \xi\left(\mathbf{E}_{n} \backslash\{e\}\right)\right) \\
& =\frac{b}{b+(1-b)(1-a) c}
\end{aligned}
$$

Similarly, we get the same conditional probability that $\eta(e)=1$ in the case $k=0$. This concludes Case 4 .

Summarizing Cases $1-4$, we have that

$$
\mu\left(\eta(e)=1 \mid \xi\left(\mathbf{E}_{n} \backslash\{e\}\right)\right)= \begin{cases}\frac{b}{b+(1-b)(1-a) c} & \text { if } K_{v} \text { and } K_{w} \text { are both infinite } \\ \frac{a}{a+(1-a)(1-c)} & \text { otherwise }\end{cases}
$$

so that $\mu$ is a random-cluster measure with

$$
p=\frac{b}{b+(1-b)(1-a) c}=\frac{b\left(1-(1-b)^{n}\right)}{b\left(1-(1-b)^{n}\right)+(1-b)\left(1-(1-b)^{n-1}\right) c}
$$

and

$$
q=\frac{\frac{b}{(1-b)(1-a) c}}{\frac{a}{(1-a)(1-c)}}=\frac{\left(1-(1-b)^{n}\right)(1-c)}{(1-b)^{n} c}
$$

It remains to show that $\mu$ is invariant under graph automorphisms $\left(M^{b, c}\right.$. is not, obviously). For this, it suffices to show that $\mu$ is invariant under those graph automorphisms $\pi$ which map $v$ on $\mathbf{0}$, where $v \in \mathbf{V}_{n}$ is a nearest neighbour of 0 . Write $\pi \mu$ for the measure on $\{0,1\}^{\mathbf{E}_{n}}$ induced by choosing a configuration according to $\mu$ and then moving the configuration according to $\pi$. What we need to show is that $\pi \mu(C)=\mu(C)$ for all cylinder events $C$; a cylinder event $C$ is an event which depends on only finitely many edges. For $w \in \mathbf{V}_{n}$, let $\partial B_{w, m}$ denote the set of vertices whose distance to $w$ is exactly $m$, and note that any finite edge-set is surrounded by $\partial B_{w, m}$ for all sufficiently large $m$. Furthermore, since $\mu$ is a random-cluster measure, the probability of a cylinder event surrounded by $\partial B_{w, m}$ is determined by the distribution of $\delta_{w}\left(\partial B_{w, m}\right) \in\{0,1\}^{\partial B_{w, m}}$ where for $u \in \partial B_{w, m}$,

$$
\delta_{w}(u)= \begin{cases}1 & \text { if } u \text { is connected to } \infty \text { without using edges inside } \partial B_{w, m}, \\ 0 & \text { otherwise }\end{cases}
$$


Hence, all we need to check is that for all $\delta^{\prime} \in\{0,1\}^{\partial B_{0, m}}$,

$$
\pi \mu\left(\delta_{0}\left(\partial B_{0, m}\right)=\delta^{\prime}\right)=\mu\left(\delta_{0}\left(\partial B_{0, m}\right)=\delta^{\prime}\right)
$$

and for this we only need to check the case $m=1$ due to the Markov chain structure of $M^{b, c}$ and the fact that when we move outwards from $v$, we also move outwards from 0 except possibly at the first step. The $m=1$ instance of (15) is verified by tedious but straightforward calculations for $M^{b, c}$.

Proof of Proposition 2.3. The extremality of $\mu$ implies that the existence of infinite clusters has probability 0 or 1 . If this probability is 0 , then we have (i) by the definition of random-cluster measures. We proceed to prove that if $\mu(\exists$ infinite clusters $)=1$, then we have (ii).

Let $\psi^{-1}:\{0,1\}^{\mathbf{E}_{n}} \rightarrow\left\{0^{\prime}, 1^{\prime}, 2^{\prime}\right\}^{\mathbf{E}_{n}}$ be the inverse mapping of $\psi$ yielding a configuration which is consistent with $M^{b, c}$ (by the second paragraph in the previous proof, this is well-defined). Pick $e \in \mathbf{E}_{n}$. What we first need to show is that any two cylinder events $C_{1}$ and $C_{2}$ defined on $\left\{e^{\prime} \in \mathbf{E}_{n}: e<e^{\prime}\right\}$ and on $\left\{e^{\prime} \in \mathbf{E}_{n}: e \nless e^{\prime}, e^{\prime} \neq e\right\}$, respectively, satisfy

$$
\mu\left(C_{1} \mid C_{2}, \psi^{-1}(\eta(e))\right)=\mu\left(C_{1} \mid \psi^{-1}(\eta(e))\right) .
$$

Let $\partial B_{0, m}$ and $\delta_{0}\left(\partial B_{0, m}\right)$ be as in the previous proof, and let $B_{0, m} \subset \mathbf{E}_{n}$ be the set of edges surrounded by $\partial B_{0, m}$. The conditional distribution of $\eta\left(B_{0, m}\right) \in$ $\{0,1\}^{B_{0, m}}$ given $\delta_{0}\left(B_{0, m}\right)$ is a finite graph random-cluster measure with edge set $B_{0, m}$ and a vertex set obtained by identifying all $u \in \partial B_{0, m}$ for which $\delta_{0}(u)=1$. Write $\mu_{m, \delta_{0}}$ for this measure.

Pick $m$ so large that $C_{1}$ and $C_{2}$ are defined in terms of $\eta\left(B_{0, m}\right)$. It follows from the consistency of conditional probabilities for random-cluster measures that

$$
\mu_{m, \delta_{0}}\left(C_{2} \mid C_{1}, \psi^{-1}(\eta(e))\right)=\mu_{m, \delta_{0}}\left(C_{2} \mid \psi^{-1}(\eta(e))\right)
$$

because $C_{2}$ can only depend on $C_{1}$ through the indicator function of the event that $e$ is connected to $\infty$ via its descendants. Hence $C_{1}$ and $C_{2}$ are conditionally independent given $\psi^{-1}(\eta(e))$ and $\delta_{0}$, so that

$$
\mu_{m, \delta_{0}}\left(C_{1} \mid C_{2}, \psi^{-1}(\eta(e))\right)=\mu_{m, \delta_{0}}\left(C_{1} \mid \psi^{-1}(\eta(e))\right)
$$

Notice that $E_{m+1, \delta_{0}}\left(\mu_{m, \delta_{0}}\left(C_{1}\right)\right)=\mu_{m+1, \delta_{0}}\left(C_{1}\right)$, where $E_{m+1, \delta_{0}}$ denotes expectation with respect to $\mu_{m+1, \delta_{0}}$, and similarly for other cylinder events. Letting $m \rightarrow \infty$ and using the backward martingale convergence theorem we obtain (16), because by extremality of $\mu$ the limiting probabilities are a.s. constants.

This shows that $\psi^{-1}(\mu)$ has a Markov chain structure. The invariance under graph automorphisms implies that the transition probabilities are the same at every $v \in \mathbf{V}_{n} \backslash\{\boldsymbol{0}\}$, and also exchangeable with respect to the children. It remains to show that the transition probabilities are exactly those given for $M^{b, c}$. We will just sketch how this is done, as the details are similar to computations we have already seen, and somewhat cumbersome to write down. We proceed as above by considering $\mu_{m, \delta_{0}}$, letting $m \rightarrow \infty$, and using the backward martingale convergence theorem. The $\mathrm{Bin}^{*}$-distribution of the number of siblings with value $2^{\prime}$ is implied by the observation that the probability that $e$ percolates outwards to infinity, given that $k(\geqq 1)$ of its siblings does, must be independent of $k$. The fact that the remaining edges get values $0^{\prime}$ and $1^{\prime}$ according 
to i.i.d. Bernoulli trials follows similarly. The value of $a$ (in terms of $b$ ) is necessary to make the ratio in (11) equal 1; otherwise $\mu\left(\eta(e)=1 \mid \eta\left(\mathbf{E}_{n} \backslash\{e\}\right)\right)$ would depend on $K_{v}$ in a way that would ruin the random-cluster structure. The value of $\theta$ for the Bin*-distribution at $\mathbf{0}$, finally, is necessary to get the right conditional probabilities in Cases 3 and 4 in the proof of Proposition 2.2 when $\mathbf{0} \in K_{y}$.

\section{Proofs of main results}

The keys to proving Proposition 1.4 and Theorems 1.5-1.7 are Propositions 2.2 and 2.3, which reduce many of our questions to that of finding $b$ and $c$ which solve (2) and (3) for given $p$ and $q$. Solving for $c$ in (2) and (3), respectively, yield

$$
c=\frac{b\left(1-(1-b)^{n}\right) \frac{1-p}{p}}{(1-b)\left(1-(1-b)^{n-1}\right)}
$$

and

$$
c=\frac{1-(1-b)^{n}}{1+(q-1)(1-b)^{n}}
$$

We can then solve for $b$ by setting the right hand sides of (17) and (18) equal. A glance at (18) reveals that once we have a solution $b \in(0,1)$, we also have $c \in(0,1)$. It turns out to be more convenient to set $x=1-b$ and solve for $x$ instead of $b$. We get

$$
f_{p, q}(x)=0,
$$

where

$$
f_{p, q}(x)=(q-1) x^{n+1}+\left(1-\frac{p}{1-p}-q\right) x^{n}+\left(\frac{p}{1-p}+1\right) x-1 .
$$

We see that $x=1$ solves (19), whence $f_{p, q}(x)$ factorizes into

$$
f_{p, q}(x)=(x-1) g_{p, q}(x),
$$

where

$$
g_{p, q}(x)=(q-1) x^{n}-\frac{p}{1-p}\left(x^{n-1}+x^{n-2}+\ldots+x\right)+1
$$

whence we may alternatively solve

$$
g_{p, q}(x)=0
$$

(note that we are not interested in the solution $x=1$ to (19), because we need to have $b$ strictly between 0 and 1 ).

Proof of Proposition 1.4. We may assume $p \in(0,1)$, since otherwise the result is trivial. Suppose first that $p(p+(1-p) q)^{-1} \leqq n^{-1}$. Then the i.i.d. measure on $\{0,1\}^{\mathbf{E}_{n}}$ with edge probability $p(p+(1-p) q)^{-1}$ is a random-cluster measure with parameters $p$ and $q$ by the observation that this measure has no infinite clusters (which follows from a branching process argument).

On the other hand, suppose $p(p+(1-p) q)^{-1}>1 / n$, so that $q<(n-1)$ $p(1-p)^{-1}$. We then have $g_{p, q}(0)=1$ and $g_{p, q}(1)=q-(n-1) p(1-p)^{-1}$ $<0$. Hence, there must be some $x \in(0,1)$ which solves $(20)$. Let $b=1-x$ 
and let $c$ be given by (18). Proposition 2.2 now tells us that $\psi\left(M^{b, c}\right)$ is a random-cluster measure with parameters $p$ and $q$.

Before proceeding with the remaining proofs, we need to give a more precise definition of the maximal random-cluster measure $\mu_{1}^{p, q}$ for $p \in[0,1]$ and $q \geqq 1$. For $S \subseteq \mathbf{E}_{n}$ and two configurations $\eta, \eta^{\prime} \in\{0,1\}^{S}$, write $\eta \leqq \eta^{\prime}$ if $\eta(e) \leqq \eta^{\prime}(e)$ for every $e \in S$. For two probability measures $\mu$ and $\mu^{\prime}$ on $\{0,1\}^{S}$, we write $\mu \leqq \mu^{\prime}$ if there exists a measure $v$ on $\{0,1\}^{S} \times\{0,1\}^{S}$ whose first and second marginals are $\mu$ and $\mu^{\prime}$ (i.e. $v$ is a coupling of $\mu$ and $\mu^{\prime}$ ) such that

$$
v\left(\left(\eta, \eta^{\prime}\right): \eta \leqq \eta^{\prime}\right)=1 \text {. }
$$

For finite $S \subset \mathbf{E}_{n}$ and two configurations $\xi$ and $\xi^{\prime}$ on $\mathbf{E}_{n} \backslash S$ such that $\xi \leqq \xi^{\prime}$ we have

$$
\mu_{S, \xi}^{p, q} \leqq \mu_{S, \xi^{\prime}}^{p, q}
$$

for $q \geqq 1$. This is well known for $\mathbf{Z}^{d}$ and can be shown either using the FKG inequality (see next Section) or by the method used to prove Lemma 2.4. Let $B_{m} \subset \mathbf{E}_{n}$ be as in the proof of Proposition 2.3, and let $\mu_{1, m}^{p, q}$ be the measure on $\{0,1\}^{\mathbf{E}_{n}}$ which assigns probability 1 to the configuration $\xi_{1} \equiv 1$ on $\mathbf{E}_{n} \backslash B_{m}$ and whose projection on $B_{m}$ is $\mu_{B_{m}, \xi_{1}}^{p, q}$. We can view $\mu_{1, m}^{p, q}$ as $\mu_{1, m+1}^{p, q}$ conditioned on all edges in $B_{m+1} \backslash B_{m}$ being present. It then follows from (21) that $\mu_{1, m+1}^{p, q} \leqq \mu_{1, m}^{p, q}$, whence by monotonicity the limiting measure

$$
\mu_{1}^{p, q}=\lim _{m \rightarrow \infty} \mu_{1, m}^{p, q}
$$

exists.

Lemma $3.1 \mu_{1}^{p, q}$ is a random-cluster measure with parameters $p$ and $q$. Moreover,

$$
\mu \leqq \mu_{1}^{p, q}
$$

for any other random-cluster measure $\mu$ with the same parameters.

Proof. We have $\mu_{1,1}^{p, q} \geqq \mu_{1,2}^{p, q} \geqq \ldots \geqq \mu_{1}^{p, q}$ whence we can find a measure $v$ on $\left(\{0,1\}^{\mathbf{E}_{n}}\right)^{\{1,2, \ldots\} \cup\{\infty\}}$ with marginals $\mu_{1,1}^{p, q}, \mu_{1,2}^{p, q}, \ldots, \mu_{1}^{p, q}$ satisfying

$$
v\left(\left(\eta_{1}, \eta_{2}, \ldots, \eta\right): \eta_{1} \geqq \eta_{2} \geqq \ldots \geqq \eta\right)=1
$$

and

$$
v\left(\left(\eta_{1}, \eta_{2}, \ldots, \eta\right): \eta(e)=\lim _{m \rightarrow \infty} \eta_{m}(e) \text { for all } e \in \mathbf{E}_{n}\right)=1 .
$$

Pick $e \in \mathbf{E}_{n}$, let the events $C_{e}$ and $-C_{e}$ be as in Lemma 2.4, and note that $\eta \in C_{e}$ if and only if $\eta_{i} \in C_{e}$ for all $i$ while on the other hand $\eta \in \neg C_{e}$ if and only if $\eta_{i} \in \neg C_{e}$ for all sufficiently large $i$ (both statements should be read $v$-a.s.). It follows that $\mu_{1}^{p, q}$ satisfies the assumptions of Lemma 2.4 and hence is a random-cluster measure. To get (23), note that $(21)$ implies that

$$
\mu \leqq \mu_{1, m}^{p, q}
$$

for all $m$. Combining this with (22) finishes the proof. $\square$ 
It follows from Lemma 3.1 that $\mu_{1}^{p, q}$ is invariant under graph automorphisms and also extremal in the set of all random-cluster measures. Hence, by Proposition 2.3, $\mu_{1}^{p, q}$ is either i.i.d. measure with edge probability $p(p+(1-p) q)^{-1}$, or equals $\psi\left(M^{b, c}\right)$ for some choice of $b$ and $c$. In the former case we have $\theta(p, q)=0$ while in the latter case $\theta(p, q)>0$.

Next, we present two easy comparison results.

Lemma 3.2 For $q \geqq 1$ and $0 \leqq p_{1} \leqq p_{2} \leqq 1$,

$$
\mu_{1}^{p_{1}, q} \leqq \mu_{1}^{p_{2}, q} \text {. }
$$

In particular, $\theta\left(p_{1}, q\right) \leqq \theta\left(p_{2}, q\right)$.

Lemma 3.3 For $q>0$ and $0 \leqq p \leqq 1$, define $r_{1}=\min \left\{p, p(p+(1-p) q)^{-1}\right\}$ and $r_{2}=\max \left\{p, p(p+(1-p) q)^{-1}\right\}$. We then have

$$
\mu_{r_{1}} \leqq \mu_{1}^{p, q} \leqq \mu_{r_{2}},
$$

where $\mu_{r_{1}}$ and $\mu_{r_{2}}$ are i.i.d. measures with edge probabilities $r_{1}$ and $r_{2}$, respectively.

We omit the proofs, which are immediate adaptions of the corresponding results on $\mathbf{Z}^{d}$ (see $[1,14]$ ).

Proof of Theorem 1.5. By Lemma 3.2, there exists $p_{c}(q) \in[0,1]$ such that $\theta(p, q)=0$ for $p<p_{c}(q)$ and $\theta(p, q)>0$ for $p>p_{c}(q)$. To show that $p_{c}(q)>0$, pick $p<n^{-1}$. Lemma 3.3 now implies that $\theta(p, q)=0$, because the critical value of independent bond percolation on $\mathbf{T}_{n}$ equals $n^{-1}$. (This in fact shows that $p_{c}(q) \geqq n^{-1}$.)

If we instead pick $p$ so large that $p(p+(1-p) q)^{-1}>n^{-1}$, (so that in other words $\left.p>q(n+q-1)^{-1}\right)$, then we have, again by Lemma 3.3, that $\theta(p, q)>0$, whence

$$
p_{c}(q) \leqq \frac{q}{n+q-1} .
$$

We go on to study what happens when $p=q(n+q-1)^{-1}$. For $1 \leqq q \leqq 2$ we get $g_{p, q}(0)=1, g_{p, q}(1)=0$ and, for all $x \in(0,1)$,

$$
\begin{aligned}
g_{p, q}^{\prime}(x) & =n(q-1) x^{n-1}-\frac{p}{1-p}\left(1+2 x+3 x^{2}+\ldots+(n-1) x^{n-2}\right) \\
& <\left(n(q-1)-\frac{p}{1-p} \frac{n(n-1)}{2}\right) x^{n-1} \\
& =n\left(\frac{q}{2}-1\right) x^{n-1} \\
& \leqq 0
\end{aligned}
$$

whence $g_{p, q}(x) \neq 0$ for all $x \in(0,1)$. Hence, (20) has no solution $x \in(0,1)$, so $\theta(p, q)=0$. Combining this with (24) gives $p_{c}(q)=q(n+q-1)^{-1}$ for $1 \leqq q \leqq 2$. 
If instead $q>2$ and $p=q(n+q-1)^{-1}$, then $g_{p, q}(0)=1$ and $g_{p, q}(1)=0$ as before, but

$$
\begin{aligned}
g_{p, q}^{\prime}(1) & =n(q-1)-\frac{p}{1-p} \frac{n(n-1)}{2} \\
& =n\left(\frac{q}{2}-1\right) \\
& >0
\end{aligned}
$$

so that $g_{p, q}(1-\varepsilon)<0$ for sufficiently small $\varepsilon>0$. Now pick $\delta>0$ so small that we still have $g_{p, q}(1-\varepsilon)<0$ if we let $p=q(n+q-1)^{-1}-\delta$. We still have $g_{p, q}(0)=1$ for this value of $p$, so (20) must then have a solution $x \in$ $(0,1-\varepsilon)$. Hence,

$$
\theta\left(\frac{q}{n+q-1}-\delta, q\right)>0
$$

so that $p_{c}(q)<q(n+q-1)^{-1}$.

Proof of Theorem 1.6. It is immediate from Lemma 3.2 that $\theta(p, q)$ is nondecreasing. The continuity for $p<p_{c}(q)$ is trivial, while the case $p \geqq p_{c}(q)$ takes some more work.

Let $\tilde{x}(p, q)$ be the smallest nonnegative $x$ which solves (19), and note that $\theta(p, q)>0$ if and only if $\tilde{x}(p, q)<1$. Given $p$ and $q$, we have that $b$ and $c$ are continuous and strictly decreasing in $x$. Furthermore, the percolation probability is continuous and strictly increasing in $b$ and $c$, whence it is also continuous and strictly decreasing in $x$. Hence, $\mu_{1}^{p, q}=\psi\left(M^{b, c}\right)$ where $b=1-\tilde{x}$ and $c$ is given by (17). Noting also that $\theta(p, q) \rightarrow 0$ as $\tilde{x} \rightarrow 1$, we have that continuity of $\theta(p, q)$ is equivalent to continuity of $\tilde{x}(p, q)$. Hence, a discontinuity can only occur when $g_{p, q}(x)$ has a local minimum or maximum for $x=\tilde{x}$. When $1 \leqq q \leqq 2$ and $p \geqq p_{c}(q)$, we have $g_{p, q}^{\prime}(x)<0$ for all $x \in(0,1)$ by $(25)$ (with the $=$ in the third line replaced by $\leqq$ ), so $\tilde{x}(p, q)$ is a continuous function of $p$, whence the same thing holds for $\theta(p, q)$. This settles the case $1 \leqq q \leqq 2$.

Moving on to the $q>2$ case, we see that $\tilde{x}(p, q)=1$ for $p<p_{c}(q)$, and $\tilde{x}(p, q)<1$ for $p=p_{c}(q)$, whence $\theta(p, q)$ is discontinuous from the left at $p=p_{c}(q)$. To see that this is the only discontinuity, note first that

$$
f_{p, q}^{\prime \prime}(x)=n x^{n-2}\left((n+1)(q-1) x+(n-1)\left(1-\frac{p}{1-p}-q\right)\right)
$$

is zero only for $x=0$ and

$$
x=\frac{(n-1)\left(q+\frac{p}{1-p}-1\right)}{(n+1)(q-1)}>0,
$$

and that $f_{p, q}^{\prime \prime}(x)$ is negative for sufficiently small $x>0$. It follows that $f_{p, q}^{\prime \prime}(x)$ is first concave and then convex on $(0, \infty)$. Since $f(0)<0$ and $f(1)=0$ we thus have that (19) (hence (20)) has at most two solutions in $(0,1)$. Since for fixed $x \in(0,1)$ and $q$ we have that $g(x)$ is strictly decreasing in $p$, there will be exactly two solutions when $p=p_{c}(q)+\varepsilon$ for sufficiently small $\varepsilon$, and $\tilde{x}\left(p_{c}(q)+\varepsilon, q\right)$ can be made arbitrarily close to $\tilde{x}\left(p_{c}(q), q\right)$ by picking $\varepsilon$ 
sufficiently small. For $p>p_{c}(q)$ we get $g_{p, q}^{\prime}(x)<0$ for $x=\tilde{x}(p, q)$, hence continuity of $\tilde{x}(p, q)$ and $\theta(p, q)$.

By the same reasoning as in the above proof, we have, for $q>0$, that $p_{c}(q)$ is the unique value of $p$ for which (19) has a double root in $(0,1)$, justifying the remark after Theorem 1.5.

Proof of Theorem 1.7. By Theorems 1.5 and 1.6 there exists, for $q>2$ and $p \geqq p_{c}(q)$, at least one random-cluster measure for which the root percolates with positive probability. If in addition we have $p \leqq q(n+q-1)^{-1}$, then the i.i.d. measure with edge probability $p(p+(1-p) q)^{-1}$ is concentrated on the event that there is no infinite cluster (by the usual branching process comparison) whence it is a random-cluster measure. We thus have at least two different random-cluster measures.

In connection with Theorem 1.7 it is also worth noting that for $p$ in the open interval $\left(p_{c}(q), q(n+q-1)^{-1}\right)$, there are in fact even two solutions to $(19)$ in $(0,1)$, so that $\psi\left(M^{b, c}\right)$ is a random-cluster measure with parameters $p$ and $q$ for two different choices of $(b, c)$ in addition to the i.i.d. measure.

Proof of Theorem 1.8. For $q \geqq 1$ and $p<p_{c}(q)$ we have that no randomcluster measure assigns positive probability to the existence of an infinite cluster, and the same thing holds when $1 \leqq q \leqq 2$ and $p=p_{c}(q)=q(n+q-$ $1)^{-1}$ by Theorem 1.6. For $q \leqq 1$ and $p \leqq q(n+q-1)^{-1}$ we are led to the same conclusion by Lemma 3.3 and a branching process comparison. In both cases we have, for any $e \in \mathbf{E}_{n}$, that the conditional probability that $e$ is present given the configuration on $\mathbf{E}_{n} \backslash\{e\}$ a.s. equals $p(p+(1-p) q)^{-1}$. Hence i.i.d. measure with edge probability $p(p+(1-p) q)^{-1}$ is the only random-cluster measure.

We end this section by proving the following result, which provides some support for Conjecture 1.9.

Proposition 3.4. For any $q>0$ and $p>q(n+q-1)^{-1}$, there is exactly one choice of $b$ and $c$ such that $\psi\left(M^{b, c}\right)$ is a random-cluster measure with parameters $p$ and $q$.

Recall that i.i.d. measure with edge probability $p(p+(1-p) q)^{-1}$ with $q \neq 1$ and $p \geqq q(n+q-1)^{-1}$ is not a random-cluster measure with parameters $p$ and $q$. Putting together this fact with Theorem 1.8 and Propositions 2.3 and 3.4 , we can rule out the possibility of having more than one random-cluster measure which is extremal and invariant under graph automorphisms whenever $q \leqq 2$ or $p \notin\left[p_{c}(q), q(n+q-1)^{-1}\right]$.

Proof of Proposition 3.4. Consider first the case $q \leqq 2$. Then $g_{p, q}(0)=1$, $g_{p, q}(1)<0$ and $g_{p, q}^{\prime}(x)<0$ for all $x \in(0,1)$ by $(25)$ (again with $\leqq$ instead of $=$ in the third line). This implies that there is exactly one $x \in(0,1)$ which solves $(20)$.

For $q>2$, we have from the proof of Theorem 1.6 that $f_{p, q}^{\prime}(x)=0$ for at most one $x \in(0,1)$. We also have $f_{p, q}(0)=-1$ and $f_{p, q}(1-\varepsilon)>0$ for all sufficiently small $\varepsilon>0$, because $g_{p, q}(1)<0$. This is sufficient to guarantee a unique $x \in(0,1)$ which solves (19). 


\section{The FKG inequality}

One of the most useful properties of random-cluster measures is the well-known FKG inequality (see e.g. [13]), which is valid if and only if $q \geqq 1$.

Let $G$ be a finite graph with edge set $E$. A function $f:\{0,1\}^{E} \rightarrow \mathbf{R}$ is called increasing if for any $\eta, \eta^{\prime} \in\{0,1\}^{E}$ such that $\eta \leqq \eta^{\prime}$ we have $f(\eta) \leqq f\left(\eta^{\prime}\right)$. Write $E_{\mu}$ for expectation with respect to a measure $\mu$. The FKG inequality asserts that for increasing $f_{1}, f_{2}:\{0,1\}^{E} \rightarrow \mathbf{R}$,

$$
E_{\mu_{p, q}^{G}}\left(f_{1} f_{2}\right) \geqq E_{\mu_{p, q}^{G}}\left(f_{1}\right) E_{\mu_{p, q}^{G}}\left(f_{2}\right)
$$

whenever $q \geqq 1$. In other words, any two increasing functions are positively correlated.

It is natural to ask whether something similar holds for the random-cluster model on $\mathbf{T}_{n}$. We restrict to functions $f:\{0,1\}^{\mathbf{E}_{n}}$ which are cylinder, i.e. which depend on finitely on finitely many edges only.

Proposition 4.1 Let $\mu$ be a random-cluster measure for $\mathbf{T}_{n}$ with parameters $p \in[0,1]$ and $q \geqq 1$. Suppose $\mu$ is extremal in the set of all random-cluster measures. Then

$$
E_{\mu}\left(f_{1} f_{2}\right) \geqq E_{\mu}\left(f_{1}\right) E_{\mu}\left(f_{2}\right)
$$

for any two increasing cylinder functions $f_{1}$ and $f_{2}$.

Proof. We proceed approximately as in the proof of Proposition 2.3. Pick $m$ so large that $f_{1}$ and $f_{2}$ are defined in terms of edges in $B_{0, m}$. Then

$$
E_{\mu}\left(f_{1} \mid \eta\left(\mathbf{E}_{n} \backslash B_{0, m}\right)\right)=E_{\mu}\left(f_{1} \mid \delta_{0}\left(\partial B_{0, m}\right)\right)
$$

and similarly for $f_{2}$ and $f_{1} f_{2}$. We have seen that the conditional measure in the right hand side is a finite graph random-cluster measure, whence by (26)

$$
E_{\mu}\left(f_{1} f_{2} \mid \delta_{0}\left(\partial B_{0, m}\right)\right) \geqq E_{\mu}\left(f_{1} \mid \delta_{0}\left(\partial B_{0, m}\right)\right) E_{\mu}\left(f_{2} \mid \delta_{0}\left(\partial B_{0, m}\right)\right)
$$

for any $\delta_{0} \in\{0,1\}^{\partial B_{0, m}}$. We also have

$$
E_{\mu}\left(E_{\mu}\left(f_{1} \mid \delta_{0}\left(\partial B_{0, m}\right)\right) \mid \delta_{0}\left(\partial B_{0, m+1}\right)\right)=E_{\mu}\left(f_{1} \mid \delta_{0}\left(\partial B_{0, m+1}\right)\right)
$$

and again similarly for $f_{2}$ and $f_{1} f_{2}$, so we get (27) by the backward martingale convergence theorem as $m \rightarrow \infty$.

Our next question is whether (27) still holds if the assumption that $\mu$ is extremal is dropped. The answer is no, and we will now show, given any $p$ and $q$ in the region $\left\{(p, q): q>2, p \in\left[p_{c}(q), q(n+q-1)^{-1}\right]\right\}$ where nonuniqueness is established, how to construct a random-cluster measure $\mu$ for which (27) fails. We will obtain $\mu$ as a convex combination $\left(\mu^{\prime}+\mu^{\prime \prime}\right) / 2$ of two other random-cluster measures $\mu^{\prime}$ and $\mu^{\prime \prime}$.

Let $e \in \mathbf{E}_{n}$ be any edge, and construct $\mu^{\prime}$ as follows. Let the descendants $\left\{e^{\prime} \in \mathbf{E}_{n}: e<e^{\prime}\right\}$ of $e$ be distributed according to the projection on $\{0,1\}^{\left\{e^{\prime} \in \mathbf{E}_{n}: e<e^{\prime}\right\}}$ of the measure on $\{0,1\}^{\mathbf{E}_{n}}$ obtained by conditioning $\mu_{1}^{p, q}$ on the event that $\eta(e)=0$. Let the rest of the edges be i.i.d. with edge probability $p(p+(1-p) q)^{-1}$. 
Let $\pi$ be a graph automorphism of $\mathbf{T}_{n}$ which maps the two endvertices of $e$ on each other, and let $\mu^{\prime \prime}=\pi \mu^{\prime}$ (i.e. $\mu^{\prime \prime}$ is the measure induced by picking a configuration according to $\mu^{\prime}$ and moving it according to $\pi$ ).

It is easy to see that $\mu^{\prime}$ is a random-cluster measure with parameters $p$ and $q$ (just check that it has the single-site conditional probabilities of Lemma $2.4)$, and that the same thing holds for $\mu^{\prime \prime}$ and $\mu=\left(\mu^{\prime}+\mu^{\prime \prime}\right) / 2$. Now let $e_{1}$ be a child of $e$, let $e_{2}$ be a sibling of $e$, and let $f_{1}=\eta\left(e_{1}\right)$ and $f_{2}=\eta\left(e_{2}\right)$. $f_{1}$ and $f_{2}$ are obviously increasing and cylinder.

We have $E_{\mu^{\prime}}\left(f_{1}\right)>E_{\mu^{\prime}}\left(f_{2}\right)$, because $\mu_{1}^{p, q}$ assigns positive probability to the event that $e$ is absent while both endvertices of $e_{1}$ are connected to infinity without using $e_{1}$. By the construction of $\mu^{\prime \prime}$, we also have that $E_{\mu^{\prime \prime}}\left(f_{1}\right)=$ $E_{\mu^{\prime}}\left(f_{2}\right)$ and $E_{\mu^{\prime \prime}}\left(f_{2}\right)=E_{\mu^{\prime}}\left(f_{1}\right)$. Furthermore, $f_{1}$ and $f_{2}$ are independent under $\mu_{1}$ (and hence under $\mu_{2}$ as well), because under $\mu_{1}, \eta\left(e_{2}\right)$ is independent of all other edges. We get

$$
\begin{aligned}
E_{\mu}( & \left.f_{1} f_{2}\right)-E_{\mu}\left(f_{1}\right) E_{\mu}\left(f_{2}\right) \\
= & \frac{1}{2}\left(E_{\mu^{\prime}}\left(f_{1} f_{2}\right)+E_{\mu^{\prime \prime}}\left(f_{1} f_{2}\right)\right)-\frac{1}{4}\left(E_{\mu^{\prime}}\left(f_{1}\right)+E_{\mu^{\prime \prime}}\left(f_{1}\right)\right)\left(E_{\mu^{\prime}}\left(f_{2}\right)+E_{\mu^{\prime \prime}}\left(f_{2}\right)\right) \\
= & \frac{1}{4}\left(E_{\mu^{\prime}}\left(f_{1}\right) E_{\mu^{\prime}}\left(f_{2}\right)+E_{\mu^{\prime \prime}}\left(f_{1}\right) E_{\mu^{\prime \prime}}\left(f_{2}\right)\right. \\
& \left.-E_{\mu^{\prime}}\left(f_{1}\right) E_{\mu^{\prime \prime}}\left(f_{2}\right)-E_{\mu^{\prime}}\left(f_{2}\right) E_{\mu^{\prime \prime}}\left(f_{1}\right)\right) \\
= & \frac{1}{4}\left(E_{\mu^{\prime}}\left(f_{1}\right) E_{\mu^{\prime}}\left(f_{2}\right)+E_{\mu^{\prime}}\left(f_{2}\right) E_{\mu^{\prime}}\left(f_{1}\right)-\left(E_{\mu^{\prime}}\left(f_{1}\right)\right)^{2}-\left(E_{\mu^{\prime}}\left(f_{2}\right)\right)^{2}\right) \\
= & -\frac{1}{4}\left(E_{\mu^{\prime}}\left(f_{1}\right)-E_{\mu^{\prime}}\left(f_{2}\right)\right)^{2} \\
< & 0
\end{aligned}
$$

so (27) fails for this choice of $\mu, f_{1}$ and $f_{2}$.

\section{How bad is the non-uniqueness?}

Once we have established non-uniqueness of random-cluster measures for certain $p$ and $q$, the next question which comes naturally to mind is: how many different random-cluster measures are there? The answer must be infinity, because whenever $\mu_{1}$ and $\mu_{2}$ are random-cluster measures with parameters $p$ and $q$ we have the same thing for any convex combination of $\mu_{1}$ and $\mu_{2}$. A more subtle issue is the number of extremal random-cluster measures. In the region of the parameter space where we have established non-uniqueness, the answer is still infinity, as stated in the following strengthening of Theorem 1.7.

Theorem 5.1 Let $q>2$. Then there is a continuum of extremal randomcluster measures for every $p$ in

$$
\left[p_{c}(q), \frac{q}{n+q-1}\right] \text {. }
$$

Proof. The idea is to generalize the construction of $\mu_{1}$ in the previous Section. Fix $p$ and $q$ in the prescribed region of the parameter space. Let $S \subset \mathbf{E}_{n}$ consist of the edges of an infinite simple path starting at $\mathbf{0}$. There are countably 
many edges $e \in \mathbf{E}_{n} \backslash S$ incident to $S$. Enumerate these edges $e_{1}, e_{2}, \ldots$ in some arbitrary way. For $i=1,2 \ldots$, let $S_{i}=\left\{e_{i}\right\} \cup\left\{e \in \mathbf{E}_{n}: e_{i}<e\right\}$, i.e. $S_{i}$ consists of $e_{i}$ and all its descendants. Note that $\left\{S, S_{1}, S_{2}, \ldots\right\}$ is a partition of $\mathbf{E}_{n}$. Now pick an arbitrary infinite sequence $B=\left(b_{1}, b_{2}, \ldots\right) \in\{0,1\}^{\mathbf{N}}$ of binary digits, and construct the random-cluster measure $\mu_{B}$ as follows.

Let all edges in $\bigcup_{i \in \mathbf{N}: b_{i}=0} S_{i}$ be i.i.d. with edge probability $p(p+(1-$ p) $q)^{-1}$. Then we want the remaining edges to be distributed according to the $\mu_{1}^{p, q}$ measure conditional on the event that $\eta\left(e_{i}\right)=0$ for all $i$ such that $b_{i}=0$. This is fine as long as the set $\left\{i: b_{i}=0\right\}$ is finite, but when this set is infinite we have some problem because we are conditioning on an event of measure 0 . To make sense of this anyway, consider the following way of constructing $\mu_{B}$ on $\mathbf{E}_{n} \backslash \bigcup_{i \in \mathbf{N}: b_{i}=0} S_{i}$. Let $\mathbf{T}_{n, B}$ be the tree obtained from $\mathbf{T}_{n}$ by "chopping off" all branches starting with an edge $e_{i}$ for which $b_{i}=0$. Now we can construct a maximal random-cluster measure for $\mathbf{T}_{n, B}$ in precisely the same manner as we did for $\mathbf{T}_{n}$ in Section 3 (strictly speaking we have not defined what a randomcluster measure on $\mathbf{T}_{n, B}$ is, but it is clear how to adapt Definition 1.3). This is tantamount to conditioning $\mu_{1}^{p, q}$ on $\eta\left(e_{i}\right)=0$ for all $i$ such that $b_{i}=0$.

As with $\mu_{1}$ in Section 4 , it is now easy to check that $\mu_{B}$ satisfies the one-dimensional conditional probabilities of Lemma 2.4 , so that it indeed is a random-cluster measure. Clearly, $\mu_{B} \neq \mu_{B^{\prime}}$ for $B \neq B^{\prime}$, so to finish the proof we just need to show that $\mu_{B}$ is extremal. Suppose $\mu_{B}=\alpha \mu^{\prime}+(1-\alpha) \mu^{\prime \prime}$ for some $\alpha \in(0,1)$ and random-cluster measures $\mu^{\prime}$ and $\mu^{\prime \prime}$. In order for $\mu^{\prime}$ and $\mu^{\prime \prime}$ to satisfy the right one-dimensional conditional probabilities, we must have, under both $\mu^{\prime}$ and $\mu^{\prime \prime}$, that each edge of $\bigcup_{i \in \mathrm{N}: b_{i}=0} S_{i}$ is independently of everything else present with probability $p(p+(1-p) q)^{-1}$. Hence it suffices to show that $\mu_{B}$ projected on $\{0,1\}^{\mathbf{E}_{n} \backslash \cup_{i \in \mathbf{N}: b_{i}=0} S_{i}}$ is an extremal random-cluster measure for the truncated tree $\mathbf{T}_{n, B}$. This, however, follows in the same way as the extremality of $\mu_{1}^{p, q}$ established in Section 3 .

Acknowledgement. I am grateful to Geoffrey Grimmett for stimulating discussions, and to the referee for careful reading and a number of valuable comments and corrections.

\section{References}

[1] Aizenman, M., Chayes, J.T., Chayes, L., Newman, C.M.: Discontinuity of the magnetization in one-dimensional $1 /|x-y|^{2}$ Ising and Potts models. J. Stat. Phys. 50, $1-40$ (1988)

[2] Benjamini, I., Peres, Y.: Markov chains indexed by trees. Ann. Probab. 22, 219-243 (1994)

[3] Bleher, P.M., Ruiz, J., Zagrebnov, V.A.: On the purity of the limiting Gibbs state for the Ising model on the Bethe lattice. J. Stat. Phys. 79, 473-482 (1995)

[4] Bollobás, B., Grimmett, G., Janson, S.: The random-cluster model on the complete graph. Probab. Theory Relat. Fields 104, 383-417 (1996)

[5] Burton, R., Keane, M.: Density and uniqueness in percolation. Comm. Math. Phys. 121, $501-505$ (1989)

[6] Chayes, J.T., Chayes, L., Sethna, J.P., Thouless, D.J.: A mean field spin glass with short range interactions. Comm. Math. Phys. 106, 41-89 (1986) 
[7] Edwards, R.G., Sokal, A.D.: Generalization of the Fortuin-Kasteleyn-Swendsen-Wang representation and Monte Carlo algorithm. Phys. Rev. D 38, 2009-2012 (1988)

[8] Fortuin, C.M.: On the random-cluster model. II. The percolation model. Physica 58, 393-418 (1972)

[9] Fortuin, C.M.: On the random-cluster model. III. The simple random-cluster process. Physica 59, 545-570 (1972)

[10] Fortuin, C.M., Kasteleyn, P.W.: On the random-cluster model. I. Introduction and relation to other models. Physica 57, 536-564 (1972)

[11] Georgii, H.-O.: Gibbs Measures and Phase Transitions. W. de Gruyter, New York (1988)

[12] Grimmett, G.: Percolation. Springer, New York (1989)

[13] Grimmett, G.: Percolative problems. Probability and Phase Transition. Kluwer, Dordrecht, (1994), pp. 69-86

[14] Grimmett, G.: The stochastic random-cluster process, and the uniqueness of randomcluster measures. Ann. Probab. (in press)

[15] Häggström, O.: Random-cluster measures and uniform spanning trees. Stoch. Proc. Appl. (in press)

[16] Higuchi, Y.: Remarks on the limiting Gibbs states on a $(d+1)$ tree. Publ. RIMS 13, 335-348 (1977)

[17] Lebowitz, J., Martin-Löf, A.: On the uniqueness of the equilibrium state for Ising spin systems. Comm. Math. Phys. 25, 276-282 (1972)

[18] Lyons, R.: The Ising model and percolation on trees and tree-like graphs. Comm. Math. Phys. 125, 337-353 (1989)

[19] Lyons, R.: Random walks and percolation on trees. Ann. Probab. 18, 931-958 (1990)

[20] Spitzer, F.: Markov random fields on an infinite tree. Ann. Probab. 3, 387-398 (1975)

[21] Zachary, S.: Countable state space Markov random fields and Markov chains on trees. Ann. Probab. 11, 894-903 (1983) 